Sādhanā Vol. 37, Part 6, December 2012, pp. 709-721. (C) Indian Academy of Sciences

\title{
An efficient similarity measure technique for medical image registration
}

\author{
VILAS H GAIDHANE ${ }^{1, *}$, YOGESH V HOTE ${ }^{2}$ and \\ VIJANDER SINGH ${ }^{1}$
}

${ }^{1}$ Department of Instrumentation and Control Engineering, Netaji Subhas Institute of Technology, University of Delhi, New Delhi, 110078 India

${ }^{2}$ Department of Electrical Engineering, Indian Institute of Technology, Roorkee, 247667 India

e-mail: vilasgd612@gmail.com; yhotefee@iitr.ernet.in

MS received 19 January 2012; revised 31 July 2012; accepted 6 September 2012

\begin{abstract}
In this paper, an efficient similarity measure technique is proposed for medical image registration. The proposed approach is based on the Gerschgorin circles theorem. In this approach, image registration is carried out by considering Gerschgorin bounds of a covariance matrix of two compared images with normalized energy. The beauty of this approach is that there is no need to calculate image features like eigenvalues and eigenvectors. This technique is superior to other wellknown techniques such as normalized cross-correlation method and eigenvalue-based similarity measures since it avoids the false registration and requires less computation. The proposed approach is sensitive to small defects and robust to change in illuminations and noise. Experimental results on various synthetic medical images have shown the effectiveness of the proposed technique for detecting and locating the disease in the complicated medical images.
\end{abstract}

Keywords. Gerschgorin circle; Gerschgorin bound; covariance matrix; eigenvalues; normalized cross-correlation; magnetic resonance images (MRI).

\section{Introduction}

Image registration is one of the fundamental tasks required in many image processing applications. It is the process of matching two data sets or images taken from the same scene recorded at different time intervals, locations, viewpoints, illuminations and sensors. It is an important process which involves four steps: feature detection, feature matching, transform model estimation

*For correspondence 
and image resampling. Particularly, image registration is required in multispectral classification, changed detection, environmental monitoring, remote sensing, geographic information system (GIS), weather forecasting, defect detection in material, computer vision and computer tomography (CT) scanning, etc. (Zitová \& Flusser 2003; Jung \& Im 2011). In recent years, due to the fast growth of advanced data acquisition systems, researchers develop a keen interest in the field of automatic image registration.

Feature matching is the main step in image registration system. In this process, the detected features in the reference image and the scene image are matched using the image pixels intensity value. There are two basic methods: area-based methods and feature-based methods which are used for image feature matching in image registration. Area-based method is called as a correlation method or template matching method. These methods use to match the windows of predefined size (smaller than the reference image) for correspondence estimation (Andronache $e t$ al 2008). If the computational speed is the main concern and images are acquired under varying conditions, then Fourier methods are more suitable for image registration. An adaptable multilayer fractional Fourier transform approach which is also a area-based method, introduced by Pan et al (2009) for image registration. Suppose, $S(x, y)$ and $R(x, y)$ are scene image and reference image, respectively, then their corresponding Fourier transforms are $S(\xi, \eta)$ and $R(\xi, \eta)$, respectively. The cross power spectrum of these two images is given as (Zitová \& Flusser 2003),

$$
\frac{S(\xi, \eta) R(\xi, \eta)}{|S(\xi, \eta) R(\xi, \eta)|}=e^{-j 2 \pi(\xi x 0-\eta y 0)}
$$

If variation in the image is present, then images can be registered using the combination of Polar-log mapping and the phase correlation (Zheng et al 2003). Viola \& Wells (1997) proposed mutual information (MI) method describes the application of the mutual information for the registration using the magnetic resonance images (MRI) and 3D object model matching. Such MI methods found applications to register MR-PET and MR-CT images of a human brain. It works with the entire data and intensity of the images. Recently, a multimodal image registration, a leading technique is introduced by Lu et al (2008) to solve medical imaging. In these techniques, mutual information method is used to measure the statistical dependencies between two images. The mutual information between two random variables $p$ and $q$ is given as (Zitová \& Flusser 2003),

$$
M(p, q)=H(p)+H(q)-H(p, q),
$$

where, $H(p)$ is the entropy of the random variables. However, these methods suffer from the problems introduced by the local noises, illuminations and deviations. To avoid these complexities, a simple and new method for medical image registration using the concept of Gerschgorin circle theorem (Gerschgorin 1931) is proposed. Recently, this theorem has been used in various engineering applications (Hote et al 2006, 2011; Hote 2009; Gaidahne et al 2012). This work has been motivated due to the need of effective matching or mismatching measure between two compared images in the presence of local distortions.

The rest of the paper is organised as follows: A brief overview of existing correlation matching methods is presented in section 2. Section 3 describes a basic Gerschgorin circle theorem and its bounds. The proposed approach for medical image registration is presented in section 4. Experimentation results and discussions are presented in section 5. Finally, conclusions are drawn in section 6 . 


\section{Normalized cross-correlation and eigenvalue-based image matching}

The similarity measure between two uncalibrated images say $R(x, y)$ and $S(x, y)$, is defined as the normalized cross-correlation which is given as (Andronache et al 2008)

$$
\mu=\frac{\sum_{x=-m}^{m} \sum_{y=-n}^{n}[R(x, y)-\bar{R}] \cdot[S(x, y)-\bar{S}]}{(2 m+1)(2 n+1) \sigma(R) \sigma(S)},
$$

where $\mu$ is the normalized cross-correlation, $\bar{R}$ and $\bar{S}$ is the average pixel value and $\sigma(R)$ and $\sigma(S)$ is the standard deviation of the elements in $R$ and $S$ images, respectively. This similarity measure is computed for windowed region pairs for which the maximum value ' 1 ' is set as matching result. For dissimilar windowed region, the value of cross-correlation also approaches to unity. Therefore, the false decision on the similarity is possible. However, if the reference image is deformed by more complex transformation, then the window is not able to match the same part of the scene image in the reference image. However, the cross-correlation method is more popular due to its simple hardware implementation, which makes useful for real time applications (Lindoso \& Entrena 2007).

Tsai \& Yang (2005) proposed an eigenvalue-based similarity measure which applied successfully for defect detection. This method is mainly responsive to local deviations in images. Moreover, this approach is based on the shape of the pair wise gray-level distribution of the two images. Suppose, $s(p, q)$ and $r(p, q)$ are the gray-levels of scene image and reference image, respectively, then the pair-wise gray values at coincident pixel locations in the $s$ and $r$ images are used as the coordinates to plot the correspondence map. For similar images, map is a straight line and it disperses away from straight line for dissimilar images. The shape of the straight line in the map is obtained from the statistical and geometrical properties of the covariance matrix which is calculated from two compared images. For any size of images, the resultant covariance matrix $\boldsymbol{A}$ is of the order $2 \times 2$ which is given as

$$
\boldsymbol{A}=\left[\begin{array}{ll}
a_{11} & a_{12} \\
a_{21} & a_{22}
\end{array}\right] .
$$

The resultant covariance matrix is positive and symmetric. It has two eigenvalues, first is called as the smallest eigenvalue $\lambda_{S}$ and second is called as the largest eigenvalue $\lambda_{L}$. Mathematically, these eigenvalues can be obtained using the elements of covariance matrix $\boldsymbol{A}$, which are given as (Tsai \& Yang 2005; Sun et al 2008),

$$
\begin{aligned}
& \lambda_{S}=\frac{1}{2}\left[a_{11}+a_{22}-\sqrt{\left(a_{11}-a_{22}\right)^{2}+4 a_{12}^{2}}\right] \\
& \lambda_{L}=\frac{1}{2}\left[a_{11}+a_{22}+\sqrt{\left(a_{11}-a_{22}\right)^{2}+4 a_{12}^{2}}\right],
\end{aligned}
$$

where $\lambda_{S} \leq \lambda_{L}$. If two compared images are identical then the shape of correspondence map is a straight line and the variation along the minor axis is zero. Thus, value of the smallest eigenvalue ideally zero if scene image and reference image is identical. The value $\lambda_{S}$ increases and becomes very large as the dissimilarity between two images increases. Thus, the similarity between reference image and scene image can be evaluated using the smallest eigenvalue. In this method, the selection of sub-image window size is an important issue. The performance of system is 
affected with small as well as the large size of sub-image window regions. This method is still superior than the area-based registration method such as normalized cross-correlation (NCC). In Normalized cross-correlation method, the value of NCC is close to ' 1 ' for two similar as well as dissimilar images. Therefore, it is very difficult to decide the similarity and dissimilarity of the images and the final decision may results into false image registration. This problem is overcome by the eigenvalue-based method (Tsai \& Yang 2005). However, it increases the burden of eigenvalues calculation. The main advantage of proposed approach is that the calculation of real eigenvalues is not required in the process. The motivation of proposed work came from the fact that the eigenvalue-based similarity measure required for image registration is calculated using the singular value decomposition (SVD), which contributes most of the computational complexity in the system (Tsai \& Yang 2005). The SVD computation is more complex due to the computation of the image covariance matrix and SVD diagonalization process. The computation of the covariance matrix requires multiplications and summation of the order $O\left(N^{3}\right)$, whereas for SVD operation, the computational complexity is of the order $O\left(N^{3}\right)$. Moreover, there are some matrices which cannot be decomposed by SVD. However, in proposed algorithm, there is no need to perform SVD decomposition. The registration of images is simply achieved by determining the Gerschgorin bound.

\section{Gerschgorin circle theorem}

Consider a matrix $\boldsymbol{A}$ such that $\boldsymbol{A}=\left(a_{i j}\right)$. The eigenvector and its respective eigenvalues are represented by $\boldsymbol{x}$ and $\lambda$, respectively. The general eigenvalue problem is represented as, $\boldsymbol{A} \boldsymbol{x}=\lambda \boldsymbol{x}$ or $|\lambda \boldsymbol{I}-\boldsymbol{A}| \boldsymbol{x}=0$. This represents $n$ simultaneous equations for the eigenvector $\boldsymbol{x}$. Moreover, the coefficients $x_{j}$ satisfies the $i^{\text {th }}$ equations as (Gerschgorin 1931; Hote 2009)

$$
\left|\left(\lambda-a_{i i}\right)\right| \leq \sum_{j \neq i}\left|a_{i j}\right|
$$

Using the above concept, Gerschgorin (1931) states that every eigenvalue of matrix $\boldsymbol{A}$ must lie inside or on the boundary of at least one of the Gerschgorin circles.

$$
\left|\lambda-a_{i i}\right| \leq \sum_{j \neq i}\left|a_{i j}\right|=A_{i} \text { for } i=\{1,2,3, \ldots, n\},
$$

where $a_{i i}$ is the center of the Gerschgorin circle with radius $A_{i}$. Then the $n$ circles are represented as

$$
\left\{\lambda\left|\lambda-a_{i i}\right|\right\}=Q_{i}
$$

Therefore, according to Gerschgorin circle theorem each eigenvalue of matrix $\boldsymbol{A}$ must lie in the union of these $n$ circles. If $\lambda(\boldsymbol{A})$ denotes the set of all eigenvalues, then

$$
\lambda(\boldsymbol{A})=\bigcup_{i=1}^{n} Q_{i}=S .
$$

In linear algebra, it is mentioned that the matrix $\boldsymbol{A}$ and $\boldsymbol{A}^{T}$ have same eigenvalues. Therefore, $\boldsymbol{A}^{T}$ generates another set say, $\boldsymbol{S}^{T}$. Then we can write

$$
\lambda(A) \in S \cap S^{T} .
$$


If matrix $\boldsymbol{A}$ and $\boldsymbol{A}^{T}$ are real symmetric matrices then,

$$
\lambda(A) \in S \text { or } S^{T} .
$$

The intersection of Gerschgorin circles gives bounds on the real axis under which eigenvalues exist. Such bounds of eigenvalues are the extreme ends of the intersection of circles which are known as Gerschgorin bounds. Suppose, the extreme left-bound is represented by $E$ and the extreme right-bound is represented by $D$ (Hote et al 2006; Hote 2009; Gaidahne et al 2012). Now, consider a $2 \times 2$ simple matrix $\boldsymbol{A}$ as,

$$
\boldsymbol{A}=\left[\begin{array}{ll}
a_{11} & a_{12} \\
a_{21} & a_{22}
\end{array}\right] .
$$

According to the Gerschgorin theorem, elements of a matrix, $a_{i i}=a_{11}$, represent the centre and $a_{i j}=a_{12}$, represent radius of the first Gerschgorin circle. Similarly, $a_{i i}=a_{22}$ represent the centre and $a_{i j}=a_{21}$, represent the radius of the second Gerschgorin circle. Thus, from first Gerschgorin circle, the left-bound can be represented as

$$
E=\left|a_{11}-a_{12}\right|
$$

Similarly, from second Gerschgorin circle, the right-bound can be represented as

$$
D=\left|a_{22}+a_{21}\right|
$$

The above Eqs. (14) and (15) play an important role in image registration application. Based on these two equations, a new approach for image registration is proposed.

\section{Proposed technique for medical image registration}

An image is a function of two real variables $x$ and $y$ with amplitude $f(x, y)$ ranges from 0 to 255 for gray-scale image. The gray scale image $f(x, y)$ is represented by $n$ rows and $m$ columns matrices. Let, $i(x, y)$ is the input image (scene image) and $r(x, y)$ is the reference image. These images may be of same size or different sizes. The covariance matrix obtained from scene image and reference image can be represented as Eq. (13). Each element of a covariance matrix can be expressed in the image pixels form as (Tsai \& Yang 2005),

$$
\begin{aligned}
a_{11} & =\left[\frac{1}{m \times n} \sum_{x=0}^{m-1} \sum_{y=0}^{n-1} i^{2}(x, y)\right]-(\bar{i})^{2}, \\
a_{22} & =\left[\frac{1}{m \times n} \sum_{x=0}^{m-1} \sum_{y=0}^{n-1} r^{2}(x, y)\right]-(\bar{r})^{2}, \\
a_{12}=a_{21}= & {\left[\frac{1}{m \times n} \sum_{x=0}^{m-1} \sum_{y=0}^{n-1} i(x, y) \cdot r(x, y)\right]-(\bar{i} \cdot \bar{r}) . }
\end{aligned}
$$

Using these elements, one can express the Gerschgorin left-bound and right-bound as 
Left-bound (from Eq. 14)

$$
E=\left[\frac{1}{m \times n} \sum_{x=0}^{m-1} \sum_{y=0}^{n-1} i^{2}(x, y)-(\bar{i})^{2}\right]-\left[\frac{1}{m \times n} \sum_{x=0}^{m-1} \sum_{y=0}^{n-1} i(x, y) \cdot r(x, y)-(\bar{i} \cdot \bar{r})\right]
$$

and Right-bound (from Eq. 15)

$$
D=\left[\frac{1}{m \times n} \sum_{x=0}^{m-1} \sum_{y=0}^{n-1} r^{2}(x, y)-(\bar{r})^{2}\right]+\left[\frac{1}{m \times n} \sum_{x=0}^{m-1} \sum_{y=0}^{n-1} i(x, y) \cdot r(x, y)-(\bar{i} \cdot \bar{r})\right] .
$$

Lemma 1 . If the input image $i(x, y)$ and reference image $r(x, y)$ are identical then the smallest eigenvalue of a covariance matrix is equal to the Gerschgorin left-bound $E$ and the largest eigenvalue is equal to the Gerschgorin right-bound D.

Proof. Let $i(x, y)=r(x, y)$ then $\bar{i}=\bar{r}$. This signifies that,

$$
\begin{gathered}
{\left[\frac{1}{m \times n} \sum_{x=0}^{m-1} \sum_{y=0}^{n-1} i^{2}(x, y)\right]-(\bar{i})^{2}=\left[\frac{1}{m \times n} \sum_{x=0}^{m-1} \sum_{y=0}^{n-1} r^{2}(x, y)\right]-(\bar{r})^{2},} \\
{\left[\frac{1}{m \times n} \sum_{x=0}^{m-1} \sum_{y=0}^{n-1} i(x, y) \cdot r(x, y)\right]-(\bar{i} \cdot \bar{r})=\left[\frac{1}{m \times n} \sum_{x=0}^{m-1} \sum_{y=0}^{n-1} r(x, y) \cdot i(x, y)\right]-(\bar{r} \cdot \bar{i}) .}
\end{gathered}
$$

Therefore, from Eqs. (16), (17) and (18), $a_{11}=a_{22}$ and $a_{12}=a_{21}$. Putting these values in Eq. (5) and (6), eigenvalues are obtained as

$$
\begin{aligned}
& \lambda_{S}=\left|a_{11}-a_{12}\right|, \\
& \lambda_{L}=\left|a_{22}+a_{21}\right| .
\end{aligned}
$$

Now, from Eqs. (14), (15), (23) and (24), the smallest eigenvalue $\lambda_{S}$ is the Gerschgorin leftbound $E$ and the largest eigenvalue $\lambda_{L}$ is the Gerschgorin right-bound $D$.

Lemma 2. If the input image $i(x, y)$ and reference image $r(x, y)$ are dissimilar, then the smallest eigenvalue of a covariance matrix is greater than Gerschgorin left-bound $E$ and the largest eigenvalue is less than Gerschgorin right-bound D.

Proof. Let $i(x, y) \neq r(x, y)$ and $\bar{i} \neq \bar{r}$, then $a_{11} \neq a_{22}$ and $a_{12}=a_{21}$. Putting these values in Eqs. (5) and (6), eigenvalues are obtained as,

$$
\begin{aligned}
& \lambda_{S}<\left|a_{11}-a_{12}\right|, \\
& \lambda_{L}>\left|a_{22}+a_{21}\right| .
\end{aligned}
$$

From Eqs. (23), (24), (25) and (26), it is proved that the Gerschgorin left-bound $E$ and Gerschgorin right-bound $D$ are directly related with the smallest eigenvalue $\lambda_{S}$ as well as the largest eigenvalue $\lambda_{L}$ of the covariance matrix. 
Using the above mathematical analysis, a new efficient approach for medical image registration is proposed which is described in the following steps:

Step 1: Load the input image $i(x, y)$ and reference image $r(x, y)$.

Step 2: Calculate the pixel size of both images.

Step 3: Convert both images into gray-level images.

Step 4: Obtain a matrix $\boldsymbol{A}$ by calculating the elements $a_{11}, a_{22}, a_{12}$ and $a_{21}$.

(a) If the input image $i(x, y)$ and the reference image $r(x, y)$ are of the same size then the matrix $\boldsymbol{A}$ can be obtained using Eq. (16)-(18).

(b) If the input image $i(x, y)$ and the reference image $r(x, y)$ are of different sizes then first consider the windowed region of both images and obtain a matrix $\boldsymbol{A}$ using Eq. (16)-(18). In this process, padding is required.

Step 5: Apply Gerschgorin theorem to matrix $\boldsymbol{A}$ and calculate left-bound $E$ and right-bound $D$ using Eq. (14) and Eq. (15).

Step 6: Analyse the value of Gerschgorin left-bound $E$.

(a) If $E=0$ or $E \leq \delta$, (where $\delta$ is a threshold value) image registration is achieved and stop the process.

(b) Otherwise, repeat the steps 4-6 for next image windowed region.

Figures 1a and $\mathrm{b}$ show the T2-weighted neuoplastic disease (brain tumor) MRI images (Johnson \& Becker 1999, The whole brain Atlas). These images are $256 \times 256$ pixels wide with 8-bit gray levels. They are used to illustrate the working of proposed algorithm. In order to visualize the brain tumor, the resulting value of Gerschgorin left-bound $E$ is used as an intensity function, where the darkness is proportional to the zero magnitude of $E$. The larger magnitude of Gerschgorin left-bound $E$ results in the more light intensity in detected image. The lighter intensity in the resulting image shows the stronger evidence of presence of brain tumor in the brain images. The mapping of such intensity function is shown in figure 1c. Figure 2 shows the Gerschgorin left-bound $E$ and right-bound $D$. For final similarity decision, only Gerschgorin left-bound $E$ is considered. It is observed from figure 2 that the Gerschgorin left-bound $E$ is more than zero i.e., $E=1$, signifies that the both images are not similar and brain image shown in figure 1 is suffering from brain tumor.

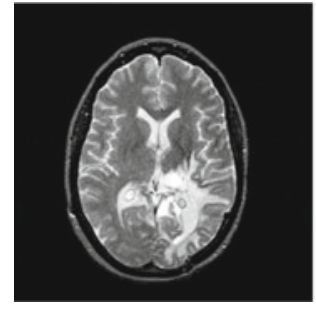

(a)

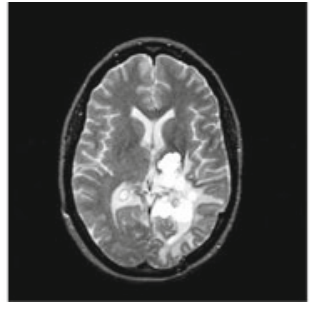

(b)

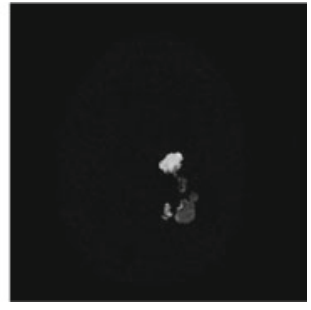

(c)

Figure 1. MRI image of brain. (a) Reference image without brain tumor, (b) image with brain tumor, and (c) brain tumor detection result. 


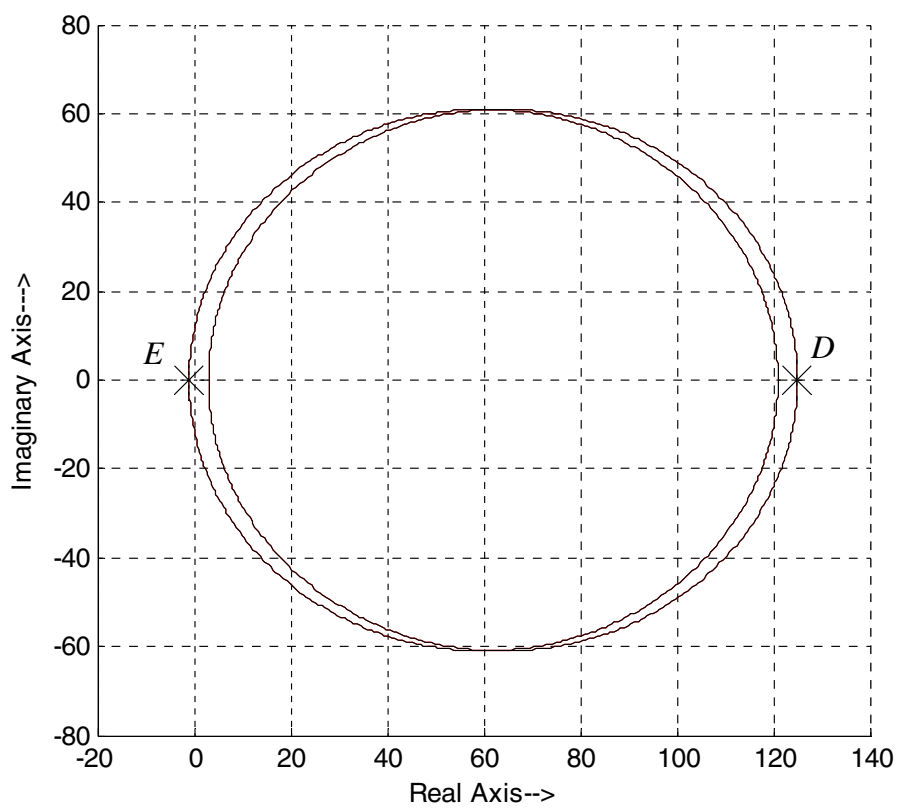

Figure 2. Gerschgorin circles, left-bound $E$ and right-bound $D$, results of figure 1a vs figure $1 \mathrm{~b}$.

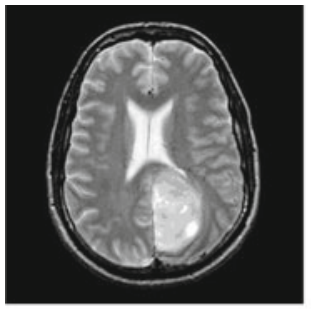

(a) $256 \times 256$

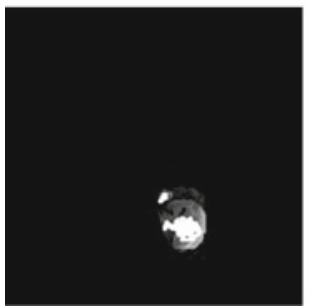

(d) $30 \times 30$

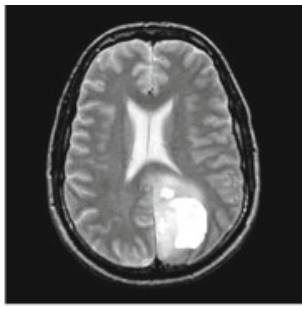

(b) $256 \times 256$

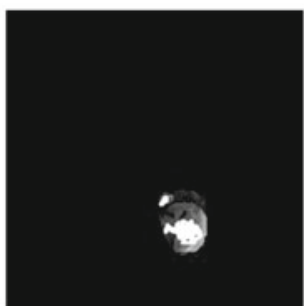

(e) $\quad 40 \times 40$

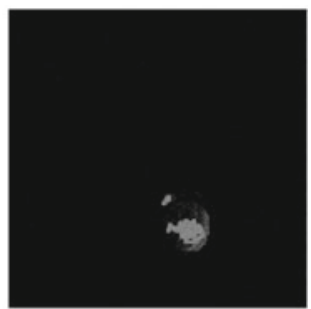

(c) $\quad 10 \times 10$

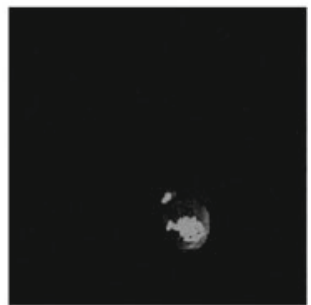

(f) $\quad 80 \times 80$

Figure 3. MRI image of brain. (a) Reference image without brain tumor, (b) image with brain tumor, and (c)-(f) brain tumor detection result for different window size. 


\section{Experimental results and discussions}

In this section, the experimental results for evaluating the efficiency of the proposed similarity measure are presented. In this experiment, our focus is on the use of proposed similarity measure technique for image matching and hence the image registration.

Test samples shown in figure 3 are used to evaluate the effect of window size considered for the image registration. Figure $3 \mathrm{a}$ shows the reference image and figure $3 \mathrm{~b}$ defective image (Glioma TITc-SPECT) (Johnson \& Becker 1999, The whole brain Atlas) of $256 \times 256$ pixels with 8-bit gray levels. Figures $3 \mathrm{c}-\mathrm{f}$ show the detection results of defective area in the image for $10 \times 10,30 \times 30,40 \times 40$ and $80 \times 80$ pixels window, respectively. The mapping of defective area shows that the small window of $10 \times 10$ pixels generates the noise in the detected image and, therefore less visible to eyes. On other hand, a window size of $80 \times 80$ pixels results into the false detection and increases the computational complexity due to the large size. Therefore, for best detection results and computational efficacy, medium window size in the range 30 to 40 pixels size is preferred.

In the next experiment, the different sizes of neighbourhood windows are used in the presence of different local distortion such as illuminations, noise and Point Spread Function (PSFs) effect to evaluate the efficiency of proposed similarity measure technique. Figure 4 shows the
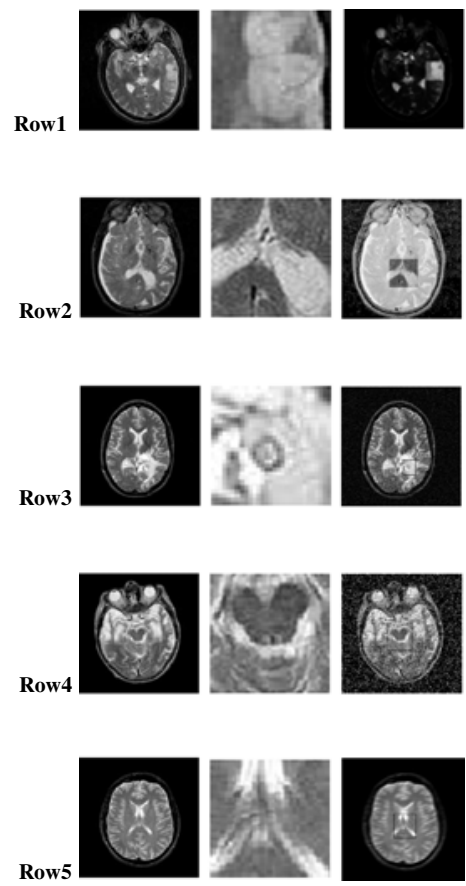

(a)

(b)

(c)
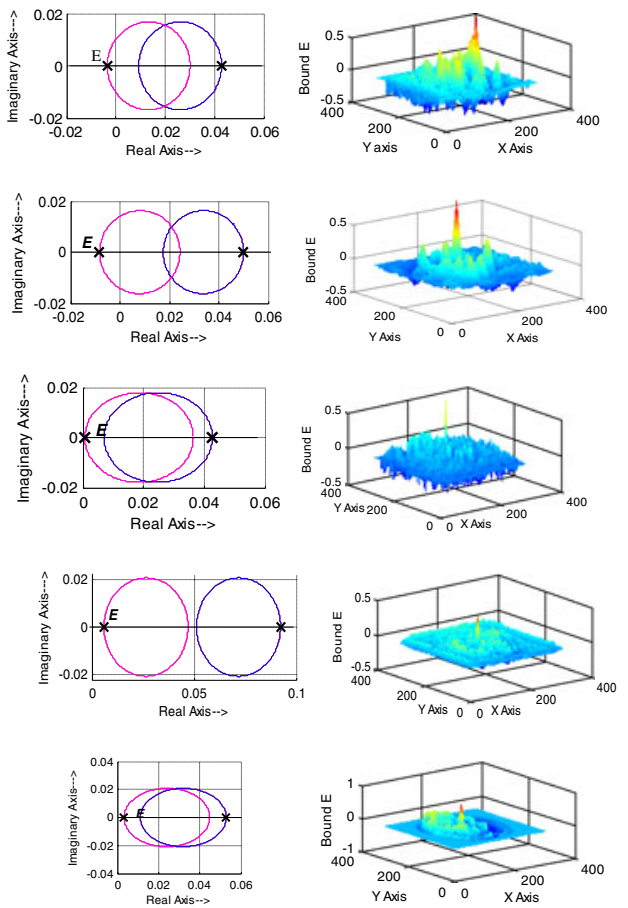

(d)

(e)

Figure 4. Image registration results of different window size template. (a) Reference image, (b) template image, (c) registration results, (d) Gerschgorin left-bound and circles, and (e) $x, y$ coordinates for registered position. 
Table 1. Gerschgorin left-bound $E$.

\begin{tabular}{lcc}
\hline Figure & Template size & Maximum value of $E$ \\
\hline Figures 1a and b & $256 \times 256$ & 1.009 \\
Figures 3a and b & $10 \times 10$ & 5.757 \\
Figures 3a and b & $30 \times 30$ & 4.755 \\
Figures 3a and b & $40 \times 40$ & 4.555 \\
Figures 3a and b & $80 \times 80$ & 5.054 \\
\hline
\end{tabular}

image registration results using different template window size in the presence of different conditions. Here, all images are taken from standard brain image dataset and the template images are obtained from the reference image by cropping the defective area using Adobe Photoshop 7.0.1 software.

In figure 4, row 1 shows the registration results for the image having the subacute stroke disease. The template image shows the particular affected region. In this experiment, the illumination (local distortion) effect having the brighten factor $\beta=-0.7$ is added in the reference image. The template of size $40 \times 40$ pixels matched with the illuminated image using proposed approach. The Gerschgorin left-bound is closer to zero value $(E=0.0049)$. Thus, the proposed approach gives the best image registration result in the presence of different illumination conditions.

Similarly, row 2 shows the registration results for the image with chronic subdural hematoma disease. The template of size $60 \times 60$ pixels is registered with the brighten image $(\beta=0.7)$. In this case, the Gerschgorin left-bound $E=0.0081$, which is nearer to zero. In row 3 , the results are shown for the image having the brain tumor. The tumor area is shown by the template image of size $30 \times 30$ pixels. The reference image is now convolved with the Gaussian noise effect (variance $v=0.01$ ). In this, the value of Gerschgorin left-bound is $E=0.0012$. Row 4 shows the registration results for Pick's disease image. The reference image is convolved with saltand-peppers noise $(d=0.02)$ and registered with the template image of size $50 \times 50$ pixels. In this case, the value of Gerschgorin left-bound is $E=0.0064$. In row 5, an AIDS dementia disease image is considered as reference image. The template image of the size $45 \times 45$ pixels is registered with the reference image which is convolved with the point spread function (PSF) effect of $h=8 \times 8$ window. In this case, the Gerschgorin left-bound is also nearer to zero ( $E=$ $0.0025)$. In these experiments, the different reference images with different illuminations, noises

Table 2. Comparison of normalized cross-correlation, eigenvalue-based similarity measure and proposed Gerschgorin left-bound $E$ with recovered $x, y$ co-ordinates.

\begin{tabular}{lccccc}
\hline Figure 4 & $\begin{array}{c}\text { Template } \\
\text { size }\end{array}$ & $\begin{array}{c}x, y \\
\text { co-ordinates }\end{array}$ & $\begin{array}{c}\text { Normalized cross- } \\
\text { correlation }(1-\mu) \\
\text { (Andronache } \\
\text { et al 2008) }\end{array}$ & $\begin{array}{c}\text { Eigenvalue-based } \\
\text { measure }\left(\lambda_{S}\right) \\
\text { (Tsai \& Yang } \\
\text { 2005) }\end{array}$ & $\begin{array}{c}\text { Gerschgorin-left } \\
\text { bound } E \\
\text { (Proposed } \\
\text { method) }\end{array}$ \\
\hline Row 1 & $40 \times 40$ & $(115,175)$ & 0.1049 & 0.1494 & 0.0049 \\
Row 2 & $60 \times 60$ & $(130,100)$ & 0.1281 & 0.3281 & 0.0081 \\
Row 3 & $30 \times 30$ & $(155,130)$ & 0.1110 & 0.1282 & 0.0012 \\
Row 4 & $50 \times 50$ & $(115,100)$ & 0.1462 & 0.4181 & 0.0064 \\
Row 5 & $45 \times 45$ & $(115,110)$ & 0.1378 & 0.2120 & 0.0025 \\
\hline
\end{tabular}




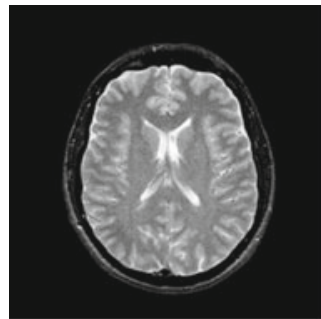

(a)

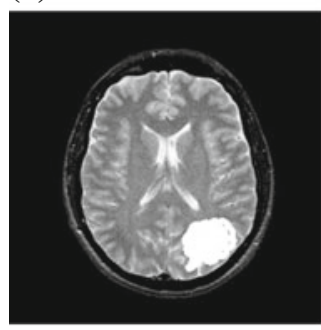

(d)

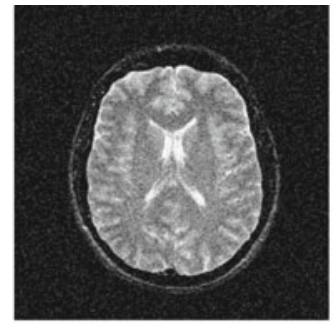

(b)

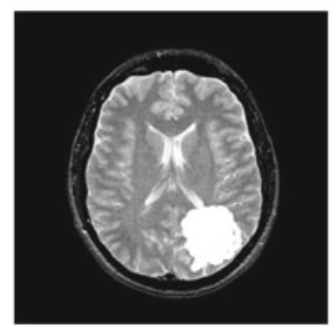

(e)

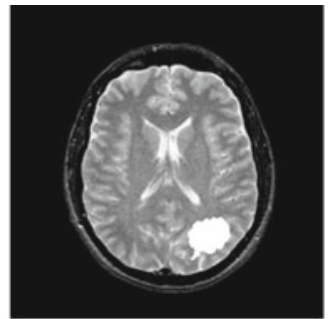

(c)

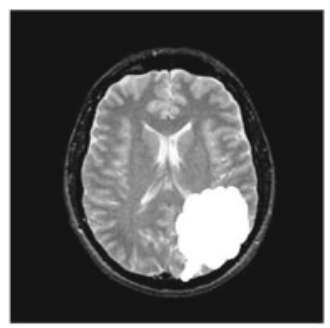

(f)

Figure 5. Brain tumor detection. (a) Reference image without brain tumor, (b) template image with Gaussian noise, (c)-(f) images with growing brain tumor.

and PSF effects are considered. In all cases, the similarity measure Gerschgorin left-bound is closer to zero, whereas the value of normalized cross-correlation and eigenvalue-based similarity measure are larger which further results into dissimilarity result. Thus, the proposed approach outperforms in the presence of different illumination, noise and PSF conditions.

Table 1 shows Gerschgorin left-bound for different windowed template images. The value of Gerschgorin left-bound $E$ should be larger for dissimilar images.

Table 2 shows the recovered $x$ and $y$ coordinates position of different windowed template images in the reference images. It also shows the comparison of normalized cross-correlation, eigenvalue-based similarity measure and proposed Gerschgorin left-bound $E$. For the perfect template matching, the value of $1-\mu, \lambda_{S}$ and Gerschgorin left-bound $E$ should be zero or nearer to zero.

From table 2, it is observed that the value of normalized cross-correlation and smallest eigenvalue-based similarity measure is greater than Gerschgorin left-bound $E$ which may result

Table 3. Comparison of normalized cross-correlation, eigenvalue-based similarity measure and proposed Gerschgorin left-bound $E$ for growing brain tumor detection.

\begin{tabular}{lccc}
\hline Figures & $\begin{array}{c}\text { Normalized cross- } \\
\text { correlation }(1-\mu)\end{array}$ & $\begin{array}{c}\text { Eigenvalue-based } \\
\text { measure }\left(\lambda_{S}\right)\end{array}$ & $\begin{array}{c}\text { Gerschgorin left-bound } E \\
\text { (Proposed approach) }\end{array}$ \\
\hline Figure 5a vs b & 0.426 & 0.612 & 0.257 \\
Figure 5a vs c & 0.259 & 0.408 & 0.478 \\
Figure 5a vs d & 0.317 & 0.637 & 0.832 \\
Figure 5a vs e & 0.426 & 0.841 & 1.143 \\
Figure 5a vs f & 0.709 & 1.275 & 2.117 \\
\hline
\end{tabular}


Table 4. Comparison of computational time and computational complexity of the normalized cross-correlation, eigenvalue-based and proposed approach.

\begin{tabular}{lccc}
\hline Similarity measure methods & $\begin{array}{c}\text { Computational time } \\
\text { (seconds) }\end{array}$ & $\begin{array}{c}\text { Computational } \\
\text { complexity }\end{array}$ & $\begin{array}{c}\text { Time saved } \\
\text { (seconds) }\end{array}$ \\
\hline Normalized cross-correlation $(\mu)$ & 0.3954 & $(255)^{3}+(255)^{2}$ & - \\
Eigenvalue-based method $\left(\lambda_{S}\right)$ & 0.3618 & $(255)^{3}+(4)^{3}$ & 0.0336 \\
Proposed approach $(E)$ & 0.2239 & $(255)^{2}+(4)$ & 0.1715 \\
\hline
\end{tabular}

into false similarity decision. This is due to the fact that the smaller eigenvalue is more affected by the noises or local disturbances.

Further, we perform the experiments to evaluate the effect of minor variations in the brain tumor size on the threshold value $\delta$ as well as the final similarity decision. In this analysis, a T2-weighted (MR-T2) healthy brain image is considered as a reference image which is shown in figure $5 \mathrm{a}$ and, figure 5b shows the noisy (Gaussian noise) test image without brain tumor. Similarly, the gradual growth of tumor is shown in figures $5 \mathrm{c}-\mathrm{f}$. In these experiments, brain tumor detection test is performed using different existing techniques as well as using proposed method. The selection of threshold value plays an important role in the final tumor detection decision. If we consider the threshold value as $\delta=0.3$, then a tumor is said to be detected only when the similarity measure value is greater than this threshold value, i.e., $\delta=0.3$. Now, we first consider the effect of noise on the final similarity decision. For this experiment, we consider figure $5 \mathrm{a}$ as a reference image and figure $5 \mathrm{~b}$ as the noisy tumor-less test image. The various values of normalized cross-correlation $(1-\mu)$, eigenvalue-based similarity measure $\left(\lambda_{S}\right)$ and Gerschgorin left-bound $(E)$ are shown in first row of table 3. From this first row, it is observed that the value of normalized cross-correlation is 0.426 and eigenvalue-based similarity measure is 0.612 which is greater than the threshold value i.e., $\delta=0.3$. These higher values show that the tumor is detected in the brain. However, it is a false decision since figure $5 \mathrm{~b}$ is without tumor. Using proposed approach, the value of Gerschgorin left-bound $E$ is 0.257 which is less than the threshold value and hence shows the tumor-less decision. From this experiment, it is proved that the proposed method is more accurate for small variations in local noise and disturbances. Further, this test for brain tumor detection is also performed for different tumors. For this experiments, the reference image, figure 5a is compared with the gradually growing brain tumor images, as shown in figures $5 \mathrm{c}-\mathrm{f}$. Similar to above experiments on tumor detection, the various similarity measures are obtained which are shown from rows 2 to 4 in table 3 . The value of similarity measures should be larger than the threshold value for correct tumor detection decision. From these rows in table 3, it is observed that the proposed Gerschgorin left-bound $E$ is greater than the threshold value as well as the normalized cross-correlation and eigenvaluebased similarity measure. This larger value of Gerschgorin bound $E$ shows the strong evidence of the brain tumor and avoids the confusion in final tumor detection decision. Thus, the proposed method is more accurate and promising good results.

All experiments are carried out on an Intel(R) Core(TM) i3 CPU with $2.4 \mathrm{GHZ}$ frequency and 4 GB RAM. The similarity measures are tested on the matrix laboratory (MATLAB) platform of version 7.0.4. Table 4 shows the comparison of computational time required by various image registration methods. Figures $5 \mathrm{a}$ and $\mathrm{c}$ of the size $256 \times 256$ pixels are considered for the calculation of computational time of various similarity measures. Moreover, the computational complexity and time saving is also summarized in table 4. 


\section{Conclusion}

In this paper, an efficient similarity measure approach is proposed for medical image registration which is based on the Gerschgorin circles theorem. The value of Gerschgorin left-bound is ideally zero for identical images and large value (more than threshold value) for dissimilar images. Experimental results have shown that the proposed similarity measure approach is superior and computationally efficient in comparison to the traditional similarity measures. Moreover, it is highly sensitive to the small defective area diagnosis and tolerable to illumination changes, noise and PSFs. This method can be useful in other medical imaging applications such as image recognition, defects detection MRI, CT scan, mismatching between fMRI and EEG/MEG images etc.

\section{References}

Andronache A, Siebenthal M V, Székely G and Cattin $\mathrm{Ph} 2008$ Non-rigid registration of multi modal images using both mutual information and cross-correlation. Med. Image Anal. 12: 3-15

Gaidahne V H, Hote Y V and Singh V 2012 Nonrigid image registration using efficient similarity measure and Levenberg-Marquardt optimization. Biomed. Eng. Lett. 2: 118-123

Gerschgorin S 1931 Ueber die abgrenzung der eigenwerte einer matrix. Izv. Akad Nauk SSSR Ser. Mat. 1: $749-754$

Hote Y V 2009 New approach of Kharitonov and Gerschgorin theorem in control system. Submitted to Delhi University

Hote Y V, Choudhury D R and Gupta J R P 2006 Gerschgorin theorem and its applications in control system problems. Proc. IEEE international conference on industrial technology 2438-2443

Hote Y V, Gupta J R P and Choudhury D R 2011 A simple approach for stability margin of discrete systems. J. Control Theory Applicat. 9: 567-570

Johnson K A and Becker J A 1999 The Whole Brain Atlas, Harvard Medical School, http://www. med.harvard.edu/aanlib

Jung Y-J and Im C-H 2011 An improved technique to consider mismatch between fMRI and EEG/MEG sources for fMRI constrained EEG/MEG source imaging. Biomed. Eng. Lett. 1: 32-41

Lindoso A and Entrena L 2007 High performance FPGA-based image correlation. J. Real-Time Image Process. 2: 223-233

Lu X, Zhang S, Su H and Chen Y 2008 Mutual information-based multimodal image registration using a novel joint histogram estimation. Comput. Med. Imaging Graphics 32: 202-209

Pan W, Qin K and Chen Y 2009 An adaptable-multilayer fractional Fourier transform approach for image registration. IEEE Trans. Pattern Anal. Mach. Intell. 31: 400-413

Sun T-H, Liu C-S and Tien F-C 2008 Invariant 2D object recognition using eigenvalues of covariance matrices, re-sampling and autocorrelation. Expert Syst. Appl. 35: 1966-1977

Tsai D-M and Yang R-H 2005 An eigenvalue-based similarity measure and its application in defect detection. Image Vision Comput. 23: 1094-1101

Viola P and Wells W M 1997 Alignment by maximization of mutual information. Int. J. Comput. Vision 24: $137-154$

Zheng D, Zhao J and Saddik A E 2003 RST-invariant digital image watermarking based on log-polar mapping and phase correlation. IEEE Trans. Circuits Syst. Video Technol. 13: 753-765

Zitová B and Flusser J 2003 Image registration methods: a survey. Image Vision Comput. 21: 977-1000 